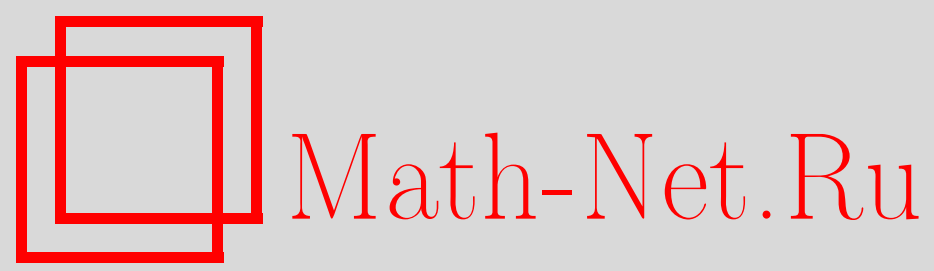

В. Н. Михно, А. С. Канарейкина, Модель формирования портфеля многопериодных инвестиций, Вестник ТвГУ. Серия: Прикладная математика, 2017, выпуск 2, 79-88

DOI: https://doi.org/10.26456/vtpmk173

Использование Общероссийского математического портала Math-Net.Ru подразумевает, что вы прочитали и согласны с пользовательским соглашением

http: //www.mathnet.ru/rus/agreement

Параметры загрузки:

IP : 34.229 .108 .108

26 апреля 2023 г., 15:28:57 
УДК $330.322(075), 519.685$

\section{МОДЕЛЬ ФОРМИРОВАНИЯ ПОРТФЕЛЯ МНОГОПЕРИОДНЫХ ИНВЕСТИЦИЙ}

Михно В.Н., Канарейкина А.С.

Тверской государственный университет, г. Тверь

Поступила в редакиию 05.04.2017, после переработки 17.05.201\%.

В статье решается задача формирования предпочтительных для инвестора портфелей многопериодных инвестиционных проектов. Актуальными вопросами в данной задаче являются ослабление условий применимости на практике математического инструментария портфельного анализа инвестиций с учетом их многопериодности. Основной целью исследования является расширение возможностей практического применения математических моделей за счет снижения требований к исходной информации относительно условий реализации проектов. Полагается, что неопределенность относительно последствий реализации проектов имеет вероятностную природу, однако вероятностные распределения последствий неизвестны. Задача ставится и решается в рамках концепции ожидаемой полезности с использованием вероятностной модели максимальной энтропии и функции полезности инвестора с постоянной несклонностью к риску, определяемой на уровнях капитализации портфелей. Приводится методика определения исходных данных для конкретизации параметров вероятностной модели, учитывающая специфику многопериодности. Решение задачи осуществляется при допущении о возможности заимствований без покрытия. При таком допущении решение о структуре портфеля выражается аналитически. Установлены области степени несклонности инвестора к риску, в которых формируемый портфель инвестора не требует осуществления заимствований без покрытия. Теоретические положения иллюстрируются примером применения модели в тестовой задаче.

Ключевые слова: инвестиционный портфель, несклонность к риску, ожидаемая полезность, многопериодная инвестиция, уровень капитализации, экстремальная энтропия.

Вестник ТвГУ. Серия: Прикладная математика. 2017. № 2. С. 79-88.

\section{Введение}

Практическое применение математических моделей портфельного анализа многопериодных инвестиционных проектов ограничено в настоящее время следующими основными обстоятельствами. Во-первых, большинство предложенных 
моделей ориентировано на однопериодный случай. В то время как многопериодность имеет свою специфику, которую невозможно учесть при непосредственном использовании или путем модификации однопериодньх моделей [1]. Во-вторых, допущения относительно используемых исходных данных, возможностей их получения и их свойств, которые берутся в основу моделей описания неопределенностей в задачах формирования оптимальных инвестиционных портфелей, являются слишком жесткими и неадекватными реальным условиям реализации многопериодных инвестиционных проектов. В-третьих, в большинстве известных моделей распределения инвестиций используемые принципы оптимальности портфелей не отражают предпочтений инвесторов, в интересах которых ставятся и решаются задачи принятия инвестиционных решений.

Перечисленные положения обуславливают актуальность задачи исследования и развития математического инструментария формирования инвестиционных портфелей на множествах многопериодных инвестиционных проектов. В качестве цели исследования следует выделить расширение возможностей практического применения математического инструментария портфельного анализа многопериодных инвестиционных проектов за счет (а) - учета специфики многопериодности реализаций инвестиций; (б) - ориентации на исходные данные и допущения, которые могут быть реально получены и отражают реальные условия в практических задачах распределения инвестиций; (в) - использования принципов оптимальности, отражающих предпочтения инвесторов, в интересах которых решаются задачи.

Для достижения перечисленных целей в работе осуществляется постановка задачи формирования инвестиционных портфелей в рамках концепции ожидаемой полезности, рассмотренная в [2] и модифицированная применительно к объектам инвестирования - многопериодным проектам. В качестве модели описания неопределенности используется, как и в [2], вероятностное распределение максимальной энтропии [3]. При этом дополнительно решается вопрос получения необходимых исходных данных в виде диапазонов возможных значений целевого показателя в каждом инвестиционном проекте. Последние оцениваются с учетом динамики реализации проектов и используются для конкретизации параметров вероятностного распределения. В качестве целевого показателя выбран уровень капитализации отдельных проектов и портфеля в целом. Функция полезности, отражающая предпочтения инвестора, конкретизируется для инвестора с постоянной несклонностью к риску. Результатом исследования являются аналитические выражения оптимального распределения инвестиций на задаваемом множестве многопериодных инвестиционных проектов. Полученные выражения могут непосредственно применяться для диверсификации инвестиций между многопериодными инвестиционными проектами. Практическое применение результатов иллюстрируется на примере решения тестовой задачи формирования портфеля многопериодных инвестиционных проектов.

\section{1. Постановка задачи}

Рассмотрим задачу распределения инвестиций на множестве $I=\{1,2 \ldots N\}$ многопериодных инвестиционных проектов [4], согласованного (распределения) с предпочтениями инвестора. Возможные распределения определим множеством 
$X=\left\{x \in R^{N}: x_{i} \geq 0, i=1, \ldots, N ; \sum_{i=1}^{N} x_{i}=1\right\}$, где $x_{i}-$ доля вложений в проект $i \in I$, а вектор $x=\left(x_{1}, \ldots x_{N}\right) \in X$ задает структуру инвестиционного портфеля. В качестве целевого показателя каждого проекта и портфеля в целом в рассматриваемой задаче используем соответственно остаточную стоимость (уровень капитализации) $C_{T}(i), i=1, \ldots, N$ (см. [4, с.214]) проекта и $C(x), x \in X$ портфеля на конец периода $T$ планирования. Целевой показатель портфеля связан с целевыми показателями отдельных проектов соотношением (см. [1])

$$
C(x)=\sum_{i=1}^{N} x_{i} C_{T}(i)
$$

Полагаем, что неопределенность условий реализации проектов имеет вероятностную природу, а $C_{T}(i), i=1, \ldots, N$ и $C(x), x \in X$ являются случайными величинами с неизвестными распределениями вероятностей. Обозначим через $P(z)$ неизвестную функцию плотности вероятностей уровня капитализации $C(x)$ портфеля $x \in X$. Пусть далее предпочтения инвестора удовлетворяют аксиомам рационального поведения и $u(C(x))$ - функция полезности [5], отражающая предпочтения инвестора на неопределенных последствиях реализации портфеля, т.е. на неопределенных значениях $C(x)$. При принятых допущениях математическая постановка задачи формирования портфеля многопериодных инвестиционных проектов осуществляется в рамках концепции ожидаемой полезности [6]:

$$
\left.\bar{u}(C(x))=\int_{-\infty}^{+\infty} u(z) P(z)\right) d z \rightarrow \max _{X}
$$

где $\bar{u}(C(x))$ - ожидаемая полезность портфеля $x \in X$

\section{2. Модель портфеля многопериодных инвестиционных проектов}

Проведем конкретизацию компонент задачи (2), необходимую для ее решения. Требуется определить вид и способ получения информации о возможных реализациях целевого показателя, конкретизировать функцию полезности инвестора и функцию плотности вероятностей значений целевого показателя.

Формальное описание многопериодных инвестиционных проектов проведем с использованием модели полного финансового плана (см. [4]). Данная модель позволяет установить для различных проектов из $I$ одинаковый (фиктивный или реальный) инвестиционный горизонт $T$. Оценивание уровней капитализации проектов и их сравнимость по данному показателю производятся с учетом осуществления при необходимости (фиктивных или реальных) дополняющих инвестиций и дополняющих заимствований в каждом периоде $t=0,1, \ldots, T$ реализации инвестиций. Теперь для оценки уровня капитализации, следуя [4, с. 208], введем дополнительно следующие обозначения: $s_{t}(i), h_{t}(i)$ - ставки по дополняющему заимствованию и дополняющей инвестиции соответственно в моменты времени от $t-1$ до $t$ для проекта $i \in I, t>0 ; z_{t}(i)$ - заданные платежи, порождаемые инвестиционным проектом $i \in I$ в моменты $t ; M_{t}$ - базовый платеж в моменты времени $t$, одинаковые для всех проектов; $Y$ - заданный, одинаковый для всех проектов уровень изъятий (дохода) с определенной структурой $f=\left(f_{0} f_{1}, \ldots, f_{T}\right)$ изъятий, при 
которой изымаемый доход в момент времени $t$ равен $f_{t} Y$. При заданных ставках $s_{t}(i), h_{t}(i), t=1, \ldots, T$ значение целевого показателя для проекта $i \in I$ вычисляется по следующему алгоритму (см. [4, с. 144]). В момент времени $t=0$ остаточная стоимость проекта равна $C_{0}(i)=M_{0}-f_{0} Y+z_{0}(i)$. Для любого момента $t=1, \ldots, T$ остаточная стоимость определяется как $C_{t}(i)=M_{t}-f_{t} Y+z_{t}(i)+\left(1+h_{t}(i)\right) C_{t-1}(i)$ при $C_{t-1}(i)>0$ и $C_{t}(i)=M_{t}-f_{t} Y+z_{t}(i)+\left(1+s_{t}(i)\right) C_{t-1}(i)$ при $C_{t-1}(i)<0$. $\mathrm{B}$ первом случае осуществляется дополняющая инвестиция в размере $C_{t-1}(i)$, а во втором - дополняющее заимствование.

Будем исходить из доступности лишь информации о пессимистичных и оптимистичных прогнозных ставках по дополняющим инвестированию и заимствованию для каждого из рассматриваемых проектов. Данная информация позволяет с использованием рассмотренного выше алгоритма получить для каждого проекта оценки возможных диапазонов $\left[C_{T}(i), \bar{C}_{T}(i)\right]$ изменения значений целевого показателя. Учитывая наличие лишь указанных данных о возможных последствиях реализации проектов, целесообразно использовать для случайных величин $C_{T}(i)$ равномерные распределения вероятностей на интервалах $\left[C_{T}(i) \bar{C}_{T}(i)\right]$ как распределения, обладающие наибольшей энтропией (см. [3]). Математические ожидания $m_{i}$ и дисперсии $\sigma_{i}^{2}$ данных распределений определяются соотношениями [7]

$$
\begin{gathered}
m_{i}=\frac{C_{T}(i)+\bar{C}_{T}(i)}{2}, \\
\sigma_{i}^{2}=\frac{\left(\overline{C_{T}}(i)-\underline{C_{T}}(i)\right)^{2}}{12}, \quad i=\overline{1, N} .
\end{gathered}
$$

Центральная предельная теорема (см. [7]) и аддитивная связь (1) целевого показателя портфеля и целевых показателей отдельных проектов позволяют принять в задаче $(2)$ в качестве функции плотности вероятностей $P(z)$ функцию $N_{m(x), \sigma^{2}(x)}(z)$ нормального распределения случайной величины $C(x)$. Для практики уже при $N \geq 3$ нормальное распределение обеспечивает приемлемую точность приближения функции $P(z)$. В предположении независимости последствий реализации различных проектов параметры $m(x), \sigma^{2}(x)$ принимаемого нормального распределения определяются соотношениями

$$
m(x)=\sum_{i=1}^{N} x_{i} m_{i} ; \sigma^{2}(x)=\sum_{i=1}^{N} x_{i}^{2} \sigma_{i}^{2} .
$$

В качестве функции полезности в (2) рассмотрим функцию, отражающую постоянную несклонность инвестора к риску (см. [5])

$$
u(C(x))=-e^{-s C(x)},
$$

где величина $s>0$ определяет степень несклонности инвестора к риску.

Доводы в пользу выбора здесь функции вида (6) полностью совпадают с обоснованиями такого выбора, приведенными в [2].

При предложенной конкретизации компонент интеграл в задаче (2) сводится к интегралу Лапласа (экспоненциальному преобразованию) [8, с. 139] для функции $N_{m(x), \sigma^{2}(x)}(z)$. Результатом такого преобразования является целевая функция в (2) вида

$$
\bar{u}(C(x))=-e^{-s m(x)+\frac{s^{2} \sigma^{2}(x)}{2}} .
$$


Предположим, что условия реализации многопериодных инвестиционных проектов допускают заимствования без покрытия (см. [4, с. 285]). Тогда с учетом (7) для решения задачи (2) применительно к многопериодным инвестициям может быть использован метод из [2]. Данный метод приводит к модели предпочтительного для инвестора портфеля многопериодных инвестиционных проектов вида

$$
x_{j}^{*}=\frac{m_{j}}{s \sigma_{j}^{2}}+\frac{1-\frac{1}{s} \sum_{i=1}^{N} \frac{m_{i}}{\sigma_{i}^{2}}}{\sigma_{j}^{2} \sum_{i=1}^{N} \frac{1}{\sigma_{i}^{2}}}, j=1, \ldots N .
$$

Укажем также, что при полной неприемлемости инвестором риска $(s=+\infty)$ оптимальная структура портфеля определяется соотношением

$$
x_{j}^{* *}=\lim _{s \rightarrow \infty} x_{j}^{*}=\frac{1}{\sigma_{j}^{2} \sum_{i=1}^{N} \frac{1}{\sigma_{i}^{2}}}, j=1, \ldots N .
$$

Заметим, что решение (8) получено при допущении о возможности заимствований без покрытия, которое снимает ограничения на неотрицательность координат вектора $x=\left(x_{1}, \ldots, x_{N}\right)$. Отклонение данного допущения не позволяет получить аналитические выражения для структуры портфеля и требует использования численных методов нелинейного программирования [9] при решении задачи (2). В этой связи целесообразно выделить критическую область значений степени $s$ несклонности инвестора к риску, в которой решение (8) удовлетворяет условиям неотрицательности. Нетрудно показать, что данная область определяется неравенством

$$
s>\max \left\{0, \max _{j}\left(\sum_{i=1}^{N} \frac{m_{i}}{\sigma_{i}^{2}}-m_{j} \sum_{i=1}^{N} \frac{1}{\sigma_{i}^{2}}\right)\right\} .
$$

Простота методики оценки индивидуальной постоянной степени несклонности к риску инвестора (см. [5]), в интересах которого решается задача, и неравенство (10) позволяют легко устанавливать приемлемость для конкретного инвестора использования аналитической модели (8) портфеля многопериодных инвестиций без принятия допущения о заимствованиях без покрытия.

\section{3. Иллюстративное применение модели}

Пусть $I=\{1,2,3\}$ - множество многопериодных инвестиционных проектов с горизонтом планирования $T=3$ года, представленных моделями полных финансовых планов в Таблице 1. Портфель формируется для инвестора с постоянной несклонностью к риску в условиях неограниченного несовершенного рынка капитала

Оптимистичные $\bar{h}_{t}, \underline{s}_{t}$ и пессимистичные $\underline{h_{t}}, \bar{s}_{t}$ прогнозы по процентным ставкам для каждого проекта представлены в Таблице 2.

Рассчитанные согласно алгоритму п.2 и формулам $(3),(4)$ диапазоны возможных значений уровней $C_{T}(i)$ капитализации проектов, их математические ожидания и дисперсии представлены в Таблице 3. 
Таблича 1: Полные финансовые планы проектов

\begin{tabular}{|l|l|l|l|l|}
\hline Момент времени $t$ & 0 & 1 & 2 & 3 \\
\hline Проект $1 z_{t}(1)$ & -500 & -400 & 800 & 400 \\
Проект 2 $z_{t}(2)$ & -300 & -350 & 1100 & -200 \\
Проект $3 z_{t}(3)$ & -900 & 800 & 360 & 100 \\
\hline Базовые платежи $M_{t}$ & 600 & 100 & -200 & 800 \\
Изъятия $f_{t} Y$ & 20 & 22 & 24 & 26 \\
\hline
\end{tabular}

Таблица 2: Прочентные ставки

\begin{tabular}{|l|l|l|l|l|l|}
\hline \multirow{2}{*}{ Проект } & \multirow{2}{*}{$t$} & \multicolumn{2}{|l|}{$\begin{array}{l}\text { Оптимистический } \\
\text { прогно3 }\end{array}$} & \multicolumn{2}{l|}{$\begin{array}{l}\text { Пессимистический } \\
\text { прогноз }\end{array}$} \\
\cline { 3 - 6 } & & $s_{t}(i)$ & $h_{t}(i)$ & $\bar{s}_{t}(i)$ & $h_{t}(i)$ \\
\hline \multirow{3}{*}{1} & 1 & 0,1 & 0,08 & 0,17 & 0,02 \\
\cline { 2 - 6 } & 2 & 0,095 & 0,079 & 0,21 & 0,022 \\
\cline { 2 - 6 } & 3 & 0,092 & 0,084 & 0,19 & 0,019 \\
\hline \multirow{3}{*}{2} & 1 & 0,01 & 0,09 & 0,17 & 0,03 \\
\cline { 2 - 7 } & 2 & 0,099 & 0,085 & 0,23 & 0,032 \\
\cline { 2 - 6 } & 3 & 0,11 & 0,089 & 0,2 & 0,04 \\
\cline { 2 - 6 } & 1 & 0,098 & 0,07 & 0,25 & 0,044 \\
\cline { 2 - 6 } & 2 & 0,09 & 0,057 & 0,21 & 0,034 \\
\cline { 2 - 6 } & 3 & 0,098 & 0,06 & 0,17 & 0,02 \\
\hline
\end{tabular}

Таблица 3: Характеристики целевых показателей проектов

\begin{tabular}{|l|l|l|l|}
\hline Инвестиционный проект & 1 & 2 & 3 \\
\hline$C_{T}(i)$ & 1479,3 & 1502,6 & 1516,9 \\
\hline$C_{T}(i)$ & 1518,7 & 1567,2 & 1608,2 \\
\hline$m_{i}$ & 1499 & 1534,9 & 1562,5 \\
\hline$\sigma_{i}^{2}$ & 129,4 & 347,2 & 695,5 \\
\hline
\end{tabular}

В Таблице 4 представлены структуры портфелей, полученные согласно (8), (9), предпочтительные для инвесторов с различными степенями постоянной несклонности к риску, а также для инвестора с полной неприемлемостью риска.

Таблица 4: Структуры портфеля

\begin{tabular}{|l|l|l|l|l|l|}
\hline$s$ & 1 & 2 & 3 & $x^{*}$ & $x^{* *}$ \\
\cline { 1 - 5 } 0.1 & $-0,607$ & 0,807 & 0,001 & $(-0,607 ; 0,807 ; 0,001)$ & \multirow{2}{*}{$(0,641 ; 0,239 ; 0,119)$} \\
\cline { 1 - 4 } 0.5 & 0,392 & 0,353 & 0,255 & $(0,392 ; 0,353 ; 0,255)$ & \\
\hline 0.9 & 0,503 & 0,302 & 0,195 & $(0,503 ; 0,302 ; 0,195)$ & \\
\hline
\end{tabular}


Из (10) следует, что в рассматриваемой иллюстративной задаче значение $s>0,19$ степени несклонности инвестора к риску определяет инвесторов, для которых выбор портфеля (8) не приводит к необходимости заимствования без покрытия. Сформированные портфели многопериодных инвестиций - это портфели с максимальной ожидаемой полезностью для инвесторов с соответствующими степенями несклонности к риску. Численные результаты имеют наглядную интерпретацию. Последнюю удобно привести с использованием классических аттестационных характеристик моделей формирования инвестиционных портфелей, а именно с позиций достигаемых ожидаемых значений $m(x)$ целевого показателя и его дисперсии $\sigma^{2}(x)$. Согласно $(5)$ указанные характеристики для полученных портфелей имеют следующие значения:

- для инвестора, полностью отвергающего (по нашей терминологии) риск, имеем $m\left(x^{* *}\right)=1513.64, \sigma^{2}\left(x^{* *}\right)=82.84$;

- для инвестора, у которого степень несклонности к риску равна $s=0.9$, имеем $m\left(x^{*}\right)=1522.22, \sigma^{2}\left(x^{*}\right)=90.85$

- для инвестора, у которого степень несклонности к риску равна $s=0.5$, имеем $m\left(x^{*}\right)=1527.55, \sigma^{2}\left(x^{*}\right)=108.34$.

Трактуя дисперсию капитализации отдельных проектов и портфеля в целом как меру риска, связанную с результатами их реализации, видим, что при возрастании степени несклонности инвестора к риску выбираются менее рискованные портфели. При этом, рост степени несклонности инвестора к риску приводит к перераспределению долей инвестиционного вложения между проектами в пользу проектов с меньшей дисперсией уровня капитализации. Снижение рискованности портфелей достигается одновременно с уменьшением ожидаемого уровня капитализации портфеля. Отмеченные свойства модели (8) портфеля многопериодных инвестиций согласуются со свойствами классических моделей. Укажем еще на отражаемый численными результатами эффект диверсификации инвестиций дисперсии (риски) выбираемых портфелей меньше дисперсий (рисков) любого из проектов анализируемого множества.

\section{Заключение}

Рассмотренная в статье модель формирования портфеля многопериодных инвестиций является обобщением на многопериодный случай подхода, связанного с описанием вероятностной неопределенности на основе критерия экстремальной энтропии. При этом методика определения необходимых для конкретизации вероятностной модели исходных данных в виде диапазонов возможных значений целевого показателя на анализируемых многопериодных инвестиционных проектах, легко реализуема в реальных задачах. Результатом исследований являются аналитические выражения для структур портфелей многопериодных инвестиционных проектов, предпочтительных для инвесторов с постоянной несклонностью к риску. Предложенную модель отличают простота и обоснованность необходимых исходных данных, простота применения модели и соответствие принимаемых допущений реальным условиям реализации многопериодных инвестиций. Указанные 
положения обуславливают более широкие возможности использования рассмотренной математической модели в практических задачах портфельного анализа многопериодных инвестиций.

\section{Список литературы}

[1] Агасандян Г.А. Элементы многопериодной портфельной модели. М.: ВЦ РАН 1997. $29 \mathrm{c}$.

[2] Михно В.Н. Модель максимальной энтропии для формирования инвестиционного портфеля // Вестник ТвГУ. Серия: Прикладная математика. 2017. № 1. C. $45-55$.

[3] Чисар И., Кернер Я. Теория информации. М.: «Мир», 1985. 400 с.

[4] Крушвиц Л. Инвестиционные расчеты. СПб.: Питер, 2001. 432 с.

[5] Кини Р.Л., Райфа Х. Принятие решений при многих критериях: предпочтения и замещения. М.: Радио и связь, 1981. 560 с.

[6] Фон Нейман Дж., Моргенштерн О. Теория игр и экономическое поведение. М.: Наука, 1970. 983 c.

[7] Крамер Г. Математические методы статистики. М.: «Мир», 1975. 648 с.

[8] Романовский П.И. Ряды Фурье. Теория поля. Аналитические и специальные функции. Преобразование Лапласа. М.: Наука, 1973. 338 с.

[9] Гилл Ф., Мюррей У., Райт М. Практическая оптимизация. М.: «Мир», 1985. $500 \mathrm{c}$.

\section{Библиографическая ссылка}

Михно В.Н., Канарейкина А.С. Модель формирования портфеля многопериодных инвестиций // Вестник ТвГУ. Серия: Прикладная математика. 2017. № 2. C. $79-88$.

\section{Сведения об авторах}

\section{1. Михно Владимир Николаевич}

заведующий кафедрой математической статистики и системного анализа Тверского государственного университета.

Россия, 170100, г. Тверь, ул. Желябова, д. З3, ТвГУ.

E-mail:vnmikhno@gmail.com

\section{2. Канарейкина Анастасия Сергеевна}

магистрант факультета прикладной математики и кибернетики Тверского государственного университета.

Россия, 170100, г. Тверь, ул. Желябова, д. З3, ТвГУ.

E-mail: anastasia06@yandex.ru 


\title{
MODEL OF FORMING MULTIPERIOD INVESTMENTS PORTFOLIO
}

\author{
Mikhno Vladimir Nikolaevich \\ Head of Mathematical Statistics and Systems Analysis department, \\ Tver State University \\ Russia, 170100, Tver, 33 Zhelyabova str., TSU. \\ E-mail:vnmikhno@gmail.com \\ Kanareikina Anastasiya Sergeevna \\ Master student of Applied Mathematics and Cybernetics faculty, \\ Tver State University \\ Russia, 170100, Tver, 33 Zhelyabova str., TSU. \\ E-mail: anastasia06@yandex.ru
}

\begin{abstract}
Received 05.04.2017, revised 17.05.201\%.
The article solves the problem of forming investor oriented portfolios of multiperiod investment projects. The problem's pertinent points include weakening the conditions of the practical applicability of the mathematical toolkit for portfolio analysis of investments considering their multiperiodity. The main goal of the research consists of broadening the possibilities of the practical application of the mathematical models at the expense of lowering requirements for the input data compared to the conditions necessary for the successful project completion. It is assumed that the uncertainty regarding the consequences of the project completion is probabilistic in nature, however, the probabilistic distributions of the consequences are unknown. The problem is set and solved within the concept of the expected utility utilizing the probabilistic model of maximum entropy and the utility function of an investor with a permanent degree of risk aversion determined at the levels of portfolio capitalization. The article provides methodology for determining input data for distilling parameters of the probabilistic model, considering the specifics of multiperiodity. The problem is solved with the assumption of the possibility of borrowing without coverage. With this assumption, the solution regarding the portfolio structure is expressed analytically. The article establishes the areas of the degree of the investor's risk aversion in which the investor portfolio that is being formed does not require borrowing without coverage. Theoretical propositions are illustrated by the example of utilizing the model in a test problem.
\end{abstract}

Keywords: investment portfolio, risk aversion, expected utility, multiperiod investment, capitalization level, extreme entropy.

\section{Bibliographic citation}

Mikhno V.N., Kanareikina A.S. Model of forming multiperiod investments portfolio. Vestnik TvGU. Seriya: Prikladnaya Matematika [Herald of Tver State University. Series: Applied Mathematics], 2017, no. 2, pp. 79-88. (in Russian) 


\section{References}

[1] Agasandyan G.A. Elementy Mnogoperiodnoi Portfelnoi Modeli [Elements of MultiPeriod Portfolio Model]. CC RAS Publ., Moscow, 1997. 29 p. (in Russian).

[2] Mikhno V.N. Maximum entropy model for forming an investment portfolio. Vestnik TvGU. Seriya: Prikladnaya Matematika [Herald of Tver State University. Series: Applied Mathematics], 2017, no. 1, pp. 45-55. (in Russian)

[3] Csiszar I., Kerner J. Teoriya Informatsii [Information Theory]. "Mir" Publ., Moscow, 1985. 400 p. (in Russian).

[4] Kruschwitz L. Investitsionnie Rascheti [Investment Calculations]. PITER Publ., Saint Petersburg, 2001. 432 p. (in Russian).

[5] Keeney R.L., Raiffa H. Prinyatie Resheniy pri Mnogikh Kriteriyakh: Predpochteniya i Zameshcheniya [Decisions with Multiple Objectives: Preferences and Value Tradeoffs]. "Radio i Svyaz" Publ., Moscow, 1981. 560 p. (in Russian).

[6] Von Neumann J., Morgenstern O. Teoriya Igr i Ekonomicheskoe Povedenie [Theory of Games and Economic behavior]. Nauka Publ., Moscow, 1970. 983 p. (in Russian).

[7] Harald Cramer. Matematicheskie Metodi Statistiki [Mathematical Methods of Statistics]. "Mir" Publ., Moscow, 1975. 648 p. (in Russian).

[8] Romanovskiy P.I. Ryadi Furje. Teoriya Polya. Analiticheskie $i$ Spetsialnie Funktsii. Preobrazovanie Laplasa. Nauka Publ., Moscow, 1973. 338 p. (in Russian).

[9] Gill Ph., Murray W., Wright M. Prakticheskaya Optimizatsiya [Practical Optimization]. "Mir" Publ., Moscow, 1985. 509 p. (in Russian). 